\title{
ESTIMACIÓN DE COSTOS ECONÓMICOS EN LA ATENCIÓN DE LA NEUMONÍA NOSOCOMIAL EN UN HOSPITAL REGIONAL PERUANO, 2009 AL 2011
}

\author{
Bernardo Dámaso-Mata ${ }^{1,2, a}$, Jesús Chirinos-Cáceres ${ }^{3, b}$, Luz Menacho-Villafuerte ${ }^{1, c}$
}

\begin{abstract}
RESUMEN
Objetivos. Estimar los costos económicos en la atención de la neumonía nosocomial al compararlo con el grupo sin neumonía nosocomial en Hospital II Huánuco EsSalud, 2009 -2011. Materiales y métodos. Evaluación económica parcial. Diseño de casos y controles pareado. Se empleó una ficha de recolección. Variable dependiente: neumonía nosocomial. Variables independientes: costos directos sanitarios, costos directos no sanitarios, costos indirectos, ocupación, comorbilidad, procedencia y grado de instrucción. Se realizó análisis bivariado. Resultados. Se identificaron 40 pares de casos y controles. Los casos estuvieron hospitalizados más de dos semanas y emplearon más de dos antibióticos. Los costos directos sanitarios asociados fueron por hospitalización, antibióticos, exámenes auxiliares, evaluaciones especializadas y otras medicaciones. Los costos directos no sanitarios y los costos indirectos asociados fueron por transporte, alimentación, alojamiento, ingresos por planilla dejados de percibir, ingresos por honorarios profesionales dejados de percibir, gastos extrainstitucionales, pago a cuidadores durante hospitalización y por telefonía. Conclusiones. Los costos directos sanitarios en neumonía nosocomial fueron más del triple, mientras los costos directos no sanitarios y costos indirectos fueron más del doble al compararlo con el grupo sin infección. Se identificaron variables con mayor impacto en los costos.
\end{abstract}

Palabras clave: Costos y análisis de costo; Asignación de costos; Economía; Costo de enfermedad (fuente: DeCS BIREME)

\section{ESTIMATION OF ECONOMIC COSTS FOR THE CARE OF PATIENTS WITH NOSOCOMIAL PNEUMONIA IN A REGIONAL PERUVIAN HOSPITAL, 2009-2011}

\begin{abstract}
Objectives. To estimate and compare the economic costs for the care of patients with and without nosocomial pneumonia at Hospital II Huánuco EsSalud during 2009-2011, in Peru. Materials and Methods. This was a partial economic evaluation of paired cases and controls. A collection sheet was used. Dependent variable: nosocomial pneumonia. Independent variables: direct health costs, direct non-health costs, indirect costs, occupation, age, comorbidities, sex, origin, and education level. A bivariate analysis was performed. Results. Forty pairs of cases and controls were identified. These patients were hospitalized for $>2$ weeks and prescribed more than two antibiotics. The associated direct health costs included those for hospitalization, antibiotics, auxiliary examinations, specialized assessments, and other medications. The direct non-health costs and associated indirect costs included those for transportation, food, housing, foregone payroll revenue, foregone professional fee revenue, extra-institutional expenses, and payment to caregivers during hospitalization and by telephone. Conclusions. The direct health costs for nosocomial pneumonia patients were more than three times and the indirect costs were more than two times higher than those for the controls. Variables with the greatest impact on costs were identified.
\end{abstract}

Keywords: Costs and cost analysis; Cost allocation; Economics; Cost of illness (source: MeSH NLM)

\section{INTRODUCCIÓN}

La infección intrahospitalaria $(\mathrm{IIH})$ es aquella contraída en el hospital en cualquier paciente internado, por una razón distinta a la que motivó su ingreso hospitalario (1). La neumonía nosocomial (NN) es la que se adquiere después de $48 \mathrm{~h}$ del ingreso al hospital (2). Hidalgo et al. en su estudio sobre la prevalencia de IIH en el Hospital

\footnotetext{
Hospital II Red Asistencial Huánuco EsSalud. Huánuco, Perú.

Facultad Medicina, Universidad Nacional Hermilio Valdizán. Huánuco, Perú.

Facultad de Salud Pública y Administración, Universidad Peruana Cayetano Heredia. Lima, Perú.

Médico internista, doctor en Medicina; ${ }^{\mathrm{b}}$ médico internista, doctor en Salud Publica; ${ }^{\mathrm{c}}$ enfermera salubrista.

Recibido: 12/10/2015 Aprobado: 06/04/2016
}

Citar como: Dámaso-Mata B, Chirinos-Cáceres J, Menacho-Villafuerte L. Estimación de costos económicos en la atención de la neumonía nosocomial en un hospital regional peruano, 2009 al 2011. Rev Peru Med Exp Salud Publica. 2016;33(2):233-40. doi: 10.17843/rpmesp.2016.332.2202 
Nacional Edgardo Rebagliati Martins EsSalud 2008, determinó que la prevalencia de infecciones hospitalarias por 100 pacientes hospitalizados fue de $7,54 \%{ }^{(3)}$.

Se define "costo" a todos los recursos sacrificados o perdidos para alcanzar un objetivo preciso ${ }^{(4)}$. Además, el concepto de costo económico se refiere al consumo de un recurso que podría utilizarse con otro fin, como el recurso ya fue consumido, la oportunidad de utilizarlo para otro propósito se perdió (costo de oportunidad) ${ }^{(4,5)}$. De acuerdo con su identificación con una enfermedad, actividad, producto o departamento, los costos pueden clasificarse como costos directos y costos indirectos ${ }^{(4,5)}$. Los costos directos son aquellos gastos atribuidos directamente a la patología que origina la enfermedad temporal o permanente ${ }^{(4,5)}$. Los costos indirectos son aquellos relacionados no directamente con la enfermedad, pero si relacionados a la pérdida de productividad, por perdida de vitalidad o vida ${ }^{(4,5)}$.

Las consecuencias económicas de las IIH se traducen en una elevada carga financiera para los establecimientos de salud y, por ende, al Hospital II Huánuco EsSalud, a consecuencia del uso de recursos materiales para el control y tratamiento de las mismas (costos directos). Asimismo, ellas representan una carga monetaria para el paciente y su familia (costos indirectos), así como alteraciones en el desenvolvimiento cotidiano de las relaciones intrafamiliares ${ }^{(6)}$. Las infecciones contraídas en el hospital, generalmente, son causadas por gérmenes multirresistentes a los antibióticos, que solo responden a los antibióticos más caros, lo cual incrementa los costos totales ${ }^{(6,7)}$.

En Latinoamérica identificamos diversos estudios, que enfocan los costos de diferentes patologías desde el punto de vista de los costos directos como los indirectos, por lo que su conocimiento es importante para valorar el impacto en el sistema de salud y en las familias afectadas ${ }^{(8,9)}$. A nivel nacional se han identificado estudios hospitalarios sobre costos directos e indirectos, pero en diferentes patologías ${ }^{(7,10,11)}$. No se han encontrado estudios nacionales sobre costos en NN en Hospitales nivel II como en Huánuco. Todos los estudios identificados, al evaluar diversas patologías emplean diferentes metodologías, dificultando la sistematización de las investigaciones de costos. Cuando un integrante de la familia desarrolla $\mathrm{NN}$, se modifica el normal desenvolvimiento del hogar, causando desembolsos económicos para la atención en salud, los cuales, aunados a todos los gastos asociados directamente con la atención y la pérdida de ingresos, puede provocar gastos aun mayores a los ingresos del hogar.

El propósito fue estimar los costos económicos en los que incurre el paciente y su familia en la atención de la $\mathrm{NN}$ al compararlo con el grupo que no desarrolla NN en el Hospital II Huánuco EsSalud del 2009 al 2011.

\section{MATERIALES Y MÉTODOS}

El tipo del estudio fue cuantitativo, el diseño de casos y controles pareados con evaluación económica parcial (análisis de costo).

El diagnóstico de NN fue clínico según los criterios de la National Healthcare Safety Network (NHSN) (Criterios radiológicos: infiltrados nuevos progresivos o persistentes, o consolidación o cavitación. Al menos uno de los siguientes: fiebre mayor de $38{ }^{\circ} \mathrm{C}$; leucocitos $<4000$ y > 12000; en > 70 años de edad, alteración del estado mental sin ninguna otra causa reconocida; y al menos dos de los siguientes: aparición de esputo, o cambios en las características del esputo; incremento en la frecuencia respiratoria o incremento en la necesidad de aspiración de secreciones; inicio de tos o empeoramiento de la misma, o disnea o taquipnea, estertores y ruidos respiratorios bronquiales, empeoramiento del intercambio gaseoso). Los pacientes con NN fueron captados en hospitalización de medicina general y en la Unidad de Vigilancia Intensiva, identificados por la búsqueda activa de los investigadores.

La unidad de análisis y observación fue un paciente con NN pareado con un paciente sin NN en el Hospital II Huánuco EsSalud del 2009 al 2011. Los casos fueron pacientes con NN y >18 años de edad. Los controles no tenían NN, pero fueron pareados con cada caso por edad ( \pm 5 años) y género. No se incluyeron pacientes $<18$ años de edad, enfermedad mental, ausencia de familiares, descompensación hemodinámica y encarcelados o prisioneros.

Para el tamaño de muestra: se empleó la fórmula para estudios de casos y controles dependientes (prueba de McNemar) (proporción de casos expuestos 70\%, proporción de controles expuestos $30 \%{ }^{(3)}$, error tipo I: $0,02$, error tipo II 0,20$)$. Se obtuvieron 40 participantes para cada grupo. El muestreo fue no probabilístico por muestreo consecutivo.

La variable dependiente fue neumonía nosocomial. Las variables independientes fueron costos directos sanitarios (costo por hospitalización, costo por antibióticos, costo por exámenes auxiliares, costo por evaluaciones especializadas y otras medicaciones, costo por atención médica después del alta hospitalaria y costo por atenciones de hemodiálisis), costos directos no sanitarios (costo por transporte, costo por alimentación, costo por alojamiento, costo por atención domiciliaria por personal de salud, costo por contratación de rehabilitador, costo por gastos extrainstitucionales, costo por cuidadores durante hospitalización, costo por telefonía celular, costo por transporte a consulta médica luego del alta hospitalaria y costo por cuidadores en domicilio) y costos indirectos (costo por ingresos por 
planilla dejados de percibir y costo por ingresos por honorarios profesionales dejados de percibir). Las variables intervinientes fueron ocupación, comorbilidad, procedencia, grado de instrucción y tipo de asegurado.

La determinación de costos fue por una evaluación económica parcial (análisis de costos) ${ }^{(4)}$. La perspectiva adoptada fue la del prestador de servicios de salud (EsSalud). Las opciones a comparar fueron los costos entre los grupos con NN y $\sin N N^{(4,12,13)}$. Se calcularon los costos directos sanitarios, costos directos no sanitarios y costos indirectos. El horizonte temporal de los costes, fue considerado hasta la semana luego del alta hospitalaria. Los precios fueron obtenidos de dos fuentes: del área de costos (dependiente de la Unidad de Finanzas-Oficina Administrativa. Toma en consideración algunos costos directos) del Hospital II de la Red Asistencial Huánuco EsSalud y los precios del mercado vigentes al 15 de enero de 2012 (según la guía para el uso del catálogo de bienes, servicios y obras del MEF - descripción de grupos y clases del rubro bienes, del Ministerio de Economía y Finanzas). La determinación de los costos se realizó mediante la metodología de microcosteo por tipo de actividad (14).

El tipo de cambio (15 enero 2012) fue de 1 (un) dólar americano $=2,70$ (dos y setenta céntimos) nuevos soles. La ficha de recolección de información, con validez del contenido (cuatro expertos) obtuvo un valor de 0,80 . En la prueba piloto, la confiabilidad con el alfa de Cronbach fue de 0,90. La evaluación entre recolectores obtuvo un índice de Kappa de 0,85. En el plan de análisis, se realizó estadística descriptiva, empleándose para el análisis inferencial la prueba t de Student para muestras dependientes de distribución paramétrica. Para distribución no normal se determinó significancia estadística con $\mathrm{chi}^{2}$, McNemar, prueba de los rangos con signo de Wilcoxon y $U$ de Mann Whitney. Se consideró un valor de $p<0,05$ como nivel de significancia estadística. Se utilizó los softwares Microsoft Excel y EPIINFO.

En las consideraciones éticas, el protocolo de investigación y el consentimiento informado fueron aprobados por los comités de investigación y de ética de la Universidad Peruana Cayetano Heredia y del Hospital II Red Asistencial de Huánuco EsSalud. Toda la información fue manejada en forma anónima y confidencial.

\section{RESULTADOS}

En la Tabla 1, se presentan las características sociodemográficas, se resalta que la mayoría provenía de Huánuco y Amarilis, además de ser pensionistas.

De los diagnósticos de ingreso fueron los más frecuentes la enfermedad cerebrovascular, la enfermedad renal
Tabla 1. Características de procedencia y aseguramiento de los pacientes con y sin neumonía nosocomial en el Hospital II Huánuco EsSalud, 2009-2011 ( $n=80$ )

\begin{tabular}{lrrrr}
\hline \multirow{2}{*}{ Características } & \multicolumn{5}{c}{ Neumonía nosocomial } \\
\cline { 2 - 5 } & Si & $\%$ & No & \multicolumn{1}{c}{$\%$} \\
\hline Procedencia & 17 & 51,5 & 16 & 48,5 \\
\hline Huánuco & 6 & 37,5 & 10 & 62,5 \\
\hline Amarilis & 6 & 100,0 & 0 & 0,0 \\
\hline Lima & 3 & 60,0 & 2 & 40,0 \\
\hline Ambo & 1 & 20,0 & 4 & 80,0 \\
\hline Tingo María & 1 & 25,0 & 3 & 75,0 \\
\hline Cerro de Pasco & 0 & 0,0 & 1 & 100,0 \\
\hline La Esperanza & 6 & 60,0 & 4 & 40,0 \\
\hline Otros & & & & \\
Tipo de asegurado & 25 & 49,02 & 26 & 50,98 \\
\hline Pensionista & 10 & 66,7 & 5 & 33,3 \\
\hline Titular & 2 & 40,0 & 3 & 60,0 \\
\hline Por viudez & 1 & 100,0 & 0 & 0,0 \\
\hline Conyugue & 2 & 25,0 & 6 & 75,0 \\
\hline Ninguno & & & &
\end{tabular}

crónica, la insuficiencia respiratoria crónica agudizada y la infección del tracto urinario. Con respecto a las comorbilidades las más frecuentes fueron la hipertensión arterial, la enfermedad cerebrovascular, la infección del tracto urinario y la arritmia cardiaca. Los cinco antibióticos más empleados fueron la ceftriaxona, ciprofloxacino, ceftazidima, clindamicina y la amikacina (Tabla 2).

En cuanto a la estancia hospitalaria, esta tuvo una mediana de tres semanas en los pacientes con neumonía y el número de antibióticos usados fue de 3,5 en los mismos pacientes, estas diferencias resultaron ser estadísticamente significativas frente a los pacientes sin neumonía nosocomial (Tabla 3).

No se encontró asociación con procedencia, diagnóstico de ingreso, estado final del paciente, número de enfermedades de ingreso, ni con las diferentes comorbilidades, ni antibiótico, excepto ceftazidima, amikacina y ciprofloxacino (Tabla 4).

En la Tabla 5 se presenta el análisis inferencial, con una mayor valorización promedio en todas las características en el grupo con la infección adquirida en el hospital. La mayoría de costos fueron estadísticamente significativos, excepto los costos por la atención médica después del alta hospitalaria, las atenciones de hemodiálisis, la atención domiciliaria por personal de salud, la contratación de rehabilitador, el transporte a consulta médica y por cuidadores en domicilio.

Al comparar el grupo con $\mathrm{NN}$ y $\sin \mathrm{NN}$, los costos directos sanitarios fueron más del triple, mientras los costos directos no sanitarios y los costos indirectos fueron alrededor del doble (Tabla 6). 
Tabla 2. Características clínicas y de tratamiento de los pacientes con y sin neumonía nosocomial en el Hospital II Huánuco EsSalud, 2009-2011 ( $n=80)$

\begin{tabular}{|c|c|c|c|c|c|}
\hline \multirow{2}{*}{ Característica } & \multicolumn{4}{|c|}{ Neumonía nosocomial } & \multirow{2}{*}{$\begin{array}{c}\text { valor } \\
p\end{array}$} \\
\hline & Si & $\%$ & No & $\%$ & \\
\hline \multicolumn{6}{|l|}{ Diagnóstico de ingreso } \\
\hline Enfermedad cerebrovascular & 13 & 61,9 & 8 & 38,1 & 0,40 \\
\hline Enfermedad renal crónica & 4 & 44,4 & 5 & 55,6 & \\
\hline Insuficiencia respiratoria crónica agudizada & 4 & 57,1 & 3 & 42,9 & \\
\hline Infección del tracto urinario & 3 & 60,0 & 2 & 60,0 & \\
\hline Neumonía adquirida en la comunidad & 3 & 60,0 & 2 & 40,0 & \\
\hline Hemorragia digestiva alta & 0 & 0,0 & 4 & 100,0 & \\
\hline Obstrucción intestinal & 2 & 50,0 & 2 & 50,0 & \\
\hline Colecistitis crónica calculosa & 1 & 33,3 & 2 & 66,7 & \\
\hline Fractura & 1 & 33,3 & 2 & 66,7 & \\
\hline Otras & 9 & 47,4 & 10 & 52,6 & \\
\hline \multicolumn{6}{|l|}{ Comorbilidades } \\
\hline Hipertensión arterial & 11 & 55,0 & 9 & 45,0 & 0,606 \\
\hline Enfermedad cerebrovascular & 4 & 44,4 & 5 & 55,6 & 1,000 \\
\hline Infección del tracto urinario & 5 & 55,6 & 4 & 44,4 & 1,000 \\
\hline Arritmia cardiaca & 5 & 62,5 & 3 & 37,5 & $0,712^{*}$ \\
\hline Diabetes mellitus & 4 & 50,0 & 4 & 50,0 & 1,000 \\
\hline Postrado crónico & 5 & 71,4 & 2 & 28,6 & $0,432^{*}$ \\
\hline Anemia & 0 & 0,0 & 6 & 100,0 & $0,026^{*}$ \\
\hline Enfermedad pulmonar intersticial difusa & 0 & 0,0 & 6 & 100,0 & $0,026^{*}$ \\
\hline Antecedente neoplasias maligna & 4 & 80,0 & 1 & 20,0 & $0,359^{*}$ \\
\hline Otras comorbilidades & 40 & 55,6 & 32 & 44,4 & \\
\hline \multicolumn{6}{|l|}{ Estado final del paciente } \\
\hline Vivo & 33 & 46,5 & 38 & 53,5 & 0,154 \\
\hline Fallecido & 7 & 77,8 & 2 & 22,2 & \\
\hline \multicolumn{6}{|l|}{ Antibióticos } \\
\hline Ceftriaxona & 23 & 54,8 & 19 & 45,2 & 0,370 \\
\hline Ciprofloxacino & 29 & 74,4 & 10 & 25,6 & 0,000 \\
\hline Ceftazidima & 30 & 83,3 & 6 & 16,7 & 0,000 \\
\hline Clindamicina & 16 & 57,1 & 12 & 42,9 & 0,348 \\
\hline Amikacina & 20 & 76,9 & 6 & 23,1 & 0,001 \\
\hline Vancomicina & 6 & 85,7 & 1 & 14,3 & 0,108 \\
\hline Imipenem & 4 & 100,0 & 0 & 0,0 & 0,116 \\
\hline Metronidazol & 3 & 75,0 & 1 & 25,0 & 0,615 \\
\hline Cefotaxima & 1 & 33,3 & 2 & 66,7 & 1,000 \\
\hline Otros & 5 & 41,7 & 7 & 58,3 & \\
\hline
\end{tabular}

${ }^{*}$ Prueba exacta de Fisher

\section{DISCUSIÓN}

El Hospital II de la Red Asistencial Huánuco forma parte del sistema del Seguro Social de Salud (EsSalud), ubicado en la región centro-oriente del Perú (sierra y selva). En este establecimiento de mediana complejidad, la NN se ubica entre las primeras causas de las IIH. Las IIH son problemas de salud pública a nivel nacional e internacional al incrementar las probabilidades de complicaciones, muertes y costos para los establecimientos de salud, la familia y la sociedad. Las IIH incrementan los costos por el mayor uso de medicamentos, exámenes auxiliares y mayor estancia hospitalaria. Estas infecciones generalmente son causadas por gérmenes multirresistentes a los antibióticos, que solo responden a los antibióticos más costosos, incrementando los gastos totales incluido el presupuesto familiar ${ }^{(6,7)}$. 
Tabla 3. Días de hospitalización y número de antibióticos, según neumonía nosocomial en el Hospital II Huánuco EsSalud, $2009-2011(n=80)$

\begin{tabular}{|c|c|c|c|}
\hline \multirow{2}{*}{ Característica } & \multicolumn{2}{|c|}{ Neumonía nosocomial } & \multirow{2}{*}{$\begin{array}{c}\text { valor } \\
p\end{array}$} \\
\hline & Si & No & \\
\hline Días de hospitalización (mediana $\pm \mathrm{RIC}$ ) & $21 \pm 14$ & $7 \pm 3$ & $0,001^{*}$ \\
\hline Número de antibióticos (media $\pm \mathrm{DE}$ ) & $3,5 \pm 1,5$ & $1,6 \pm 1,1$ & $0,001 \dagger$ \\
\hline
\end{tabular}

* $U$ de Mann Whitney $\dagger \mathrm{t}$ de Student

RIC: rango intercuartil DE: desviación estandar

En nuestro estudio, los antibióticos más utilizados fueron ceftriaxona, ciprofloxacino, ceftazidima, clindamicina y amikacina. El estudio de Inan et al., demostró que los costos diarios por los antibióticos empleados fueron un elemento de gastos extraordinarios, pero que con un uso racional podría disminuir la carga financiera al establecimiento (15). Encontramos en nuestra investigación que los antibióticos fueron un componente importante de la estructura de los costos directos.

En el análisis inferencial con la NN fueron significativos los días de hospitalización, el número de antibióticos, empleo de ceftazidima, uso de amikacina y empleo de ciprofloxacino. Masterton et al. (2), Hoo et al. (16), Piskin et al. (17) y Dallas \& Kollef ${ }^{(18)}$, recomiendan el uso empírico de antibióticos al inicio del tratamiento, para luego ser específicos, esta elección influye en el pronóstico de vida. Encontramos estudios que coinciden con la mayor estancia hospitalaria y su mayor frecuencia de adultos mayores para las $N N{ }^{(6,19)}$. Estas semejanzas con nuestro estudio podrían deberse a que las IIH son patologías complejas que demandan mayores recursos humanos y económicos para los establecimientos, comprometiendo con mayor frecuencia a los adultos mayores, quienes presentan mayores riesgos de infección. A nivel latinoamericano y nacional se presenta una transición demográfica y epidemiológica que está modificando la distribución de los grupos etarios hacia una población más añosa.

Al evaluar los promedios de los costos directos sanitarios, estos fueron mayores en el grupo con NN. El estudio de Rodríguez-Burbano et al. coincidió con nuestros hallazgos ${ }^{(20)}$, sin embargo, esta publicación es parcial, al considerar solo los costos directos de la atención. El diseño más aceptado en la actualidad para estimar costos directos, es el tipo comparativo entre pacientes con y sin infección (de preferencia pareados), de tal manera que se pueda calcular el exceso atribuible a la infección ${ }^{(21)}$, metodología aplicada en nuestro estudio.

En el análisis inferencial de los costos directos no sanitarios y costos indirectos, el grupo con NN presentó los valores más elevados, resultando significativos. El estudio de Çakır-Edis et al. presentó resultados semejantes a nuestros hallazgos ${ }^{(22)}$. Lenz-Alcayaga establece una metodología cuantitativa de la determinación de los costos de tan solo aquellos en que las intervenciones difieren; su ventaja consiste en reducir el trabajo de análisis de costos, pero sin afectar la calidad de la evaluación (23).

Los costos directos sanitarios totales, en el grupo con NN, ascendieron a cerca de S/ 340 mil nuevos soles, mientras en el grupo sin la patología fue más de S/ 105 mil nuevos soles. La NN representa para el hospital, en costos, un poco más del triple comparado con aquellos que no presentan esta patología. Morano et al. revelan que la IIH produce una prolongación de la estancia hospitalaria y una mayor carga económica farmacéutica ${ }^{(24)}$. Brenner et al. demuestran mayor número de días de estancia hospitalaria y mayor gastos por uso de antibióticos ${ }^{(25)}$. La NN incrementa significativamente los costos hospitalarios.

En cuanto a los costos directos no sanitarios y costos indirectos totales, el grupo con NN presentó un monto acumulado cercano a S/ 240 mil nuevos soles, mientras

Tabla 4. Evaluación de antibióticos según neumonía nosocomial en el Hospital II Huánuco EsSalud, 2009-2011 (n=80)

\begin{tabular}{|c|c|c|c|c|c|c|c|}
\hline \multirow{2}{*}{ Antibióticos } & & \multicolumn{4}{|c|}{ Neumonía nosocomial } & \multirow{2}{*}{ OR (IC 95\%) } & \multirow{2}{*}{$\underset{p}{\text { valor }}$} \\
\hline & & Sí & $\%$ & No & $\%$ & & \\
\hline \multirow{2}{*}{ Ceftazidima } & Sí & 30 & 83,3 & 6 & 16,7 & $17,0(5,52-52,36)$ & 0,001 \\
\hline & No & 10 & 22,7 & 34 & 77,3 & & \\
\hline \multirow{2}{*}{ Amikacina } & Sí & 20 & 76,9 & 6 & 23,1 & $5,7(1,95-16,46)$ & 0,001 \\
\hline & No & 20 & 37,0 & 34 & 63,0 & & \\
\hline \multirow{2}{*}{ Ciprofloxacino } & Sí & 29 & 74,4 & 10 & 25,6 & $7,9(2,92-21,43)$ & 0,001 \\
\hline & No & 11 & 26,8 & 30 & 73,2 & & \\
\hline
\end{tabular}


Tabla 5. Análisis inferencial de los costos según el diagnóstico de neumonía nosocomial en el Hospital II Huánuco EsSalud, 2009-2011 $(n=80)$

\begin{tabular}{|c|c|c|c|}
\hline \multirow{2}{*}{ Costo por actividades (nuevos soles) } & \multicolumn{2}{|c|}{ Neumonía nosocomial } & \multirow{2}{*}{$\begin{array}{c}\text { valor } \\
p^{*}\end{array}$} \\
\hline & Sí & No & \\
\hline \multicolumn{4}{|l|}{ Costos directos sanitarios (mediana $\pm \mathrm{RIC}$ ) } \\
\hline Hospitalización & $1050 \pm 700$ & $350 \pm 150$ & 0,00 \\
\hline Antibióticos & $2730 \pm 1785$ & $420 \pm 427$ & 0,00 \\
\hline Exámenes auxiliares & $900 \pm 360$ & $470 \pm 380$ & 0,00 \\
\hline Evaluaciones especializadas y otras medicaciones & $2647 \pm 168$ & $902 \pm 95$ & 0,00 \\
\hline Atención medica después del alta hospitalaria & $100 \pm 60$ & $100 \pm 70$ & 0,252 \\
\hline Atenciones de hemodiálisis & $1760 \pm 660$ & $660 \pm 660$ & 0,317 \\
\hline \multicolumn{4}{|l|}{ Costos directos no sanitarios (mediana $\pm \mathrm{RIC}$ ) } \\
\hline Transporte & $361 \pm 262$ & $108 \pm 113$ & 0,00 \\
\hline Alimentación & $965 \pm 700$ & $287 \pm 245$ & 0,00 \\
\hline Alojamiento & $1028 \pm 750$ & $308 \pm 278$ & 0,00 \\
\hline Atención domiciliaria por personal de salud & $700 \pm 324$ & $700 \pm 467$ & 0,179 \\
\hline Contratación de rehabilitador & $327 \pm 0$ & $210 \pm 113$ & 0,379 \\
\hline Gastos extra institucionales & $414 \pm 382$ & $171 \pm 167$ & 0,00 \\
\hline Cuidadores durante hospitalización & $942 \pm 722$ & $377 \pm 180$ & 0,00 \\
\hline Por telefonía celular & $83 \pm 56$ & $25 \pm 20$ & 0,00 \\
\hline Transporte a consulta medica & $8 \pm 4$ & $8 \pm 8$ & 0,447 \\
\hline Cuidadores en domicilio & $408 \pm 326$ & $560 \pm 362$ & 0,655 \\
\hline \multicolumn{4}{|l|}{ Costos indirectos (mediana $\pm R I C$ ) } \\
\hline Ingresos por planilla dejados de percibir & $717 \pm 350$ & $350 \pm 105$ & 0,00 \\
\hline Ingresos por honorarios profesionales & $800 \pm 375$ & $480 \pm 325$ & 0,018 \\
\hline
\end{tabular}

* Prueba de los rangos con signo de Wilcoxon RIC: rango intercuartil

en el grupo sin la patología fue de más de $\mathrm{S} / 115$ mil nuevos soles. La valoración de los costos también puede ser evaluado desde la perspectiva social, ideales pero de difícil sistematización y ejecución (26). Cusmano et al. determinaron el costo social en familias con un miembro con tuberculosis a partir de tres dimensiones: costos intangibles, costos indirectos y costos directos ${ }^{(27)}$. En nuestro estudio, los costos directos no sanitarios y los costos indirectos, asumidos por la familia fueron superiores en el grupo con NN comparado con los que no tenían NN, superándolo en más del doble del monto monetario.

La diferencia encontrada entre los costos directos sanitarios, mayores a los asumidos por las familias (costos directos no sanitarios y costos indirectos), no coinciden con la bibliografía revisada. Esta divergencia podría deberse a la mayor complejidad de la NN, que demandó en el corto plazo, mayores recursos económicos al establecimiento, mientras la población asegurada más afectada fueron los pensionistas, en quienes el lucro cesante es menor comparado con otros grupos etarios (jóvenes o adultos) ${ }^{(28)}$. Además, los ancianos son más propensos a complicaciones hospitalarias. Manuele describe la relación entre los costos indirectos sobre los directos, con resultados muy variados (4:1, 6:1, 10:1 o mayor), probablemente debido a diferentes metodologías, pero, además, por cambios en los costos directos sanitarios al considerar otros costos (costos médicos por empleo de nuevos exámenes auxiliares, indemnizaciones, etc.), incluidos en la última década (29). Manuele propone que un estudio riguroso probablemente encontraría un valor menor de 1. Otra explicación de las diferencias de precio, puede ser resultado de imperfecciones del mercado o la intervención del gobierno ${ }^{(30)}$.

Una limitación que se presentó durante el desarrollo del estudio fue la concurrencia con la epidemia de la gripe A H1N1, lo que prolongó el periodo de recolección de datos, ya que toda infección respiratoria grave era considerada como primera posibilidad una infección viral. El no pareo según la enfermedad y la severidad de la enfermedad pudo originar sesgos de selección. 
Tabla 6. Costos totales según el diagnóstico de neumonía nosocomial en el Hospital II Huánuco EsSalud, 2009-2011 (n=80)

\begin{tabular}{|c|c|c|}
\hline \multirow{2}{*}{ Costo por actividades (nuevos soles) } & \multicolumn{2}{|c|}{ Neumonía nosocomial } \\
\hline & Sí & No \\
\hline \multicolumn{3}{|l|}{ Costos directos sanitarios } \\
\hline Hospitalización & 45000,00 & 16350,00 \\
\hline Antibióticos & 137900,00 & 18785,00 \\
\hline Exámenes auxiliares & 33500,00 & 18340,00 \\
\hline Evaluaciones especializadas y otras medicaciones & 107035,00 & 39315,00 \\
\hline Atención medica después del alta hospitalaria & 4680,00 & 5220,00 \\
\hline Atenciones de hemodiálisis & 9680,00 & 7260,00 \\
\hline Costos directos sanitarios total & 337795,00 & 105270,00 \\
\hline Costo total por individuo & 8444,88 & 2631,75 \\
\hline \multicolumn{3}{|l|}{ Costos directos no sanitarios } \\
\hline Transporte & 16715,00 & 5405,50 \\
\hline Alimentación & 38472,50 & 13301,00 \\
\hline Alojamiento & 41205,00 & 14130,00 \\
\hline Atención domiciliaria por personal de salud & 4526,67 & 4203,33 \\
\hline Contratación de rehabilitador & 326,67 & 1153,33 \\
\hline Gastos extra institucionales & 16578,30 & 9645,80 \\
\hline Cuidadores durante hospitalización & 45043,33 & 17626,67 \\
\hline Por telefonía celular & 3551,00 & 1117,50 \\
\hline Transporte a consulta medica & 100,00 & 136,00 \\
\hline Cuidadores en domicilio & 19856,67 & 23636,67 \\
\hline \multicolumn{3}{|l|}{ Costos indirectos } \\
\hline Ingresos por planilla dejados de percibir & 35871,28 & 16326,76 \\
\hline Ingresos por honorarios profesionales & 17175,00 & 9237,33 \\
\hline Costos directos no sanitarios e indirectos totales & 239421,42 & 115919,89 \\
\hline Costo total individual & 5985,54 & 2898,00 \\
\hline
\end{tabular}

Tipo de cambio (enero 2012) 1 dólar = 2,70 nuevos soles

En nuestra investigación, algunos costos directos sanitarios no han sido considerados, como los costos por tratamiento de otras comorbilidades, los costos por cultivos o los costos por procedimientos, pero que a pesar de estas limitaciones, nuestros datos proveen un estimado aproximado del gasto de recursos asumidos. Nuestro estudio, al emplear la metodología de evaluación económica parcial, es uno de los primeros a nivel del interior del país, permitiendo a los decisores tomar las medidas más adecuadas.

Concluimos, entonces, que los costos directos sanitarios en el grupo con infección fueron más del triple al compararlos con el grupo sin NN, mientras los costos directos no sanitarios y costos indirectos fueron más del doble al compararlo con el grupo sin infección.
El costo de la hospitalización, el costo por uso de antibióticos, el costo por exámenes auxiliares, el costo por evaluaciones especializadas y otras medicaciones fueron significativos en el grupo con NN.

Contribuciones de autoría: BDM y JCC han participado en la concepción y diseño del artículo, la recolección de datos, su redacción y aprobación de la versión final. Además, LMV realizó el Aporte de pacientes, material de estudio, obtención de financiamiento y asesoría técnica y administrativa. BDM realizó la redacción del artículo, el análisis e interpretación de datos, además de la asesoría estadística.

Fuentes de financiamiento: recurso propio de los autores.

Conflictos de interés: ninguno.

\section{REFERENCIAS BIBLIOGRÁFICAS}

1. Ducel G, FabryJ y Nicolle L. Prevención de las infecciones nosocomiales. Guía práctica. $2^{\text {a }}$ ed. Malta: OPS/OMS; 2003.

2. Masterton R, Galloway A, French G, Street M, Armstrong J, Brown E, et al. Guidelines for the management of hospital-acquired pneumonia in the UK: Report of the Working Party on Hospital-Acquired Pneumonia of the British Society for Antimicrobial Chemotherapy. J Antimicrob Chemother. 2008;62(1):5-34. doi: $10.1093 / \mathrm{jac} / \mathrm{dkn} 162$.
3. Hidalgo L, Marroquín J, Antigoni J y Samalvides F. Prevalencia de infecciones hospitalarias en un hospital peruano de nivel IV, en el año 2008. Rev Med Hered. 2011;22(2):76-81.

4. Drummond M. Métodos para la Evaluación Económica de los 
Programas de Asistencia Sanitaria. 2a ed. Madrid: Ediciones Díaz de Santos, SA; 2001.

5. OPS/OMS. Alcance y aplicación de la Economía de la Salud. Serie: Desarrollo de la Representación de la OPS/OMS en Cuba. No.24-2001. La Habana: OPS/OMS; 2001.

6. Salvatierra-González R. Costo de la infección nosocomial en nueve países de América Latina. Washington: OPS/ OMS; 2003.

7. Martínez D, Samalvides F, Valverde V, Gotuzzo E, Ganoso O y Tomateo D. El Impacto de las Neumonías Intrahospitalarias en el Servicio de Medicina del Hospital Nacional Cayetano Heredia. Enfermedades del Tórax. 2003;46(2):98-112.

8. Rocha-García A, Hernández-Peña $P$, Ruiz-Velazco S, Ávila-Burgos L, MarínPalomares T, Lazcano-Ponce E. Gasto de hogares durante la hospitalización de menores derechohabientes, con diagnóstico de leucemia, en dos hospitales en México. Salud Publica Mex. 2003;45(4):285-92.

9. Rangel-Frausto MS, Morales-García D, Báez-Martínez R, Ibarra-Blancas J, Ponce De León-Rosales S. Validación de un Programa de Vigilancia de Infecciones Nosocomiales. Salud Publica Mex. 1999;41 (Supl 1):59-63.

10. Bambaren C. Características epidemiológicas y económicas de los casos de accidentes de tránsito atendidos en el Hospital Nacional Cayetano Heredia. Rev Med Hered. 2004;15(1):30-36.

11. Modesto J, Morales A, Cabanillas O, Díaz C. Impacto económico de la peste bubónica en Cajamarca-Perú. Rev Peru Med Exp Salud Publica. 2002;19(2):74-82.

12. López Bastida J, Oliva J, Antoñanzas F, García-Altes A, Gisbert R, Mar J, et al. Propuesta de guía para la evaluación económica aplicada a las tecnologías sanitarias. Gac Sanit. 2010;24(2):15470. doi: 10.1016/j.gaceta.2009.07.011

13. García-Altés A, Navas E, Soriano MJ. Evaluación económica de intervenciones de salud pública. Gac
Sanit. 2011;25 Supl 1:25-31. doi: 10.1016/S0213-9111(11)70005-X.

14. Puig-Junoy J, Ortún-Rubio V, PintoPrades JL. ABC en Evaluación Económica: Los costes en la evaluación económica de tecnologías sanitarias. Aten Primaria. 2001;27(3):186-9.

15. Inan D, Saba R, Gunseren F, Ongut G, Turhan O, Yalcin AN, et al. Daily antibiotic cost of nosocomial infections in a Turkish university hospital. BMC Infect Dis. 2005;5:5.

16. Soo Hoo GW, Wen YE, Nguyen TV, Goetz MB. Impact of Clinical Guidelines in the Management of Severe Hospital-Acquired Pneumonia. Chest. 2005;128(4):2778-87.

17. Piskin N, Aydemir H, Oztoprak N, Akduman D, Comert F, Kokturk $\mathrm{F}$, et al. Inadequate treatment of ventilator-associated and hospitalacquired pneumonia: Risk factors and impact on outcomes. BMC Infect Dis. 2012;12:268. doi: 10.1186/14712334-12-268.

18. Dallas J, Kollef M. Severe HospitalAcquired Pneumonia: A Review for Clinicians. Curr Infect Dis Rep. 2009;11(5):349-56.

19. Sopena N, Sabria M, Neunos 2000 Study Group. Multicenter Study of HospitalAcquired Pneumonia in Non-ICU Patients. Chest. 2005;127(1):213-9.

20. Rodríguez-Burbano L, De La Hoz FP, Leal-Castro AL. Costo de neumonía nosocomial no asociada a ventilación en el Hospital Universitario de Santander 2007-2009. Rev Salud Publica (Bogota). 2013;15(2):196-207.

21. Secretaría Distrital de Salud de Bogotá. Capítulo 8. Guías para la prevención, control y vigilancia epidemiológica de infecciones [internet]. Bogotá D.C.: Secretaría Distrital de Salud de Bogotá; 2004 [citado en 22 de abril de 2016]. Disponibleen: http://www.saludcapital. gov.co/sitios/VigilanciaSaludPublica/ Todo\%20IIH/008\%20Costos.pdf

22. Çakır-Edis E, Hatipoğlu ON, Yilmam İ, Süt N. Economic burden of nosocomial pneumonia in nonintensive care clinics. Tuberk Toraks. 2015;63(1):8-12.
23. Lenz-Alcayaga R. Análisis de costos en evaluaciones económicas en salud: Aspectos introductorios. Rev Med Chile 2010;138(Supl 2):88-92.

24. Morano Amado LE, Del Campo Pérez V, López Miragaya I, Martínez Velásquez MJ, Vázquez Alvarez $\mathrm{O}$, Pedreira Andrade JD. Bacteriemia nosocomial en el paciente adulto. Estudio de costes asociados. Rev Clin Esp. 2002;202(9):476-84.

25. Brenner-Pola F, Mercelles P, Pohlenz M, Otaíza F, Alumnos del Magister en Infecciones Intrahospitalarias. Costo de las infecciones intrahospitalarias en hospitales chilenos de alta y mediana complejidad. Rev Chil infectol. 2003;20(4):285-90.

26. Loza C, Castillo-Portilla M, Rojas JL, Huayanay L. Principios básicos y alcances metodológicos de las evaluaciones económicas en salud. Rev Peru Med Exp Salud Publica. 2011;28(3):518-27.

27. Cusmano L, Morua S, Logran M, Pereyra M, Palmero D. Estudio de los costos intangibles y de la participación del componente familiar en el costo social de la Tuberculosis. Revista Argentina de Salud Pública. 2009;1(1):18-23.

28. Lim SC, Doshi V, Castasus B, Lim JK, K Mamun K. Factors Causing Delay in Discharge of Elderly Patients in an Acute Care Hospital. Ann Acad Med Singapore. 2006;35(1):27-32.

29. Manuele F. Accident Costs: Rethinking ratios of indirect to direct costs. Professional Safety. 2011; 56(1):39-47.

30. Mejía A. Evaluación económica de programas y servicios de salud. Rev Gerenc Polit Salud. 2008;7(15):91-113.

Correspondencia: Bernardo C. Dámaso Mata Dirección: Jr. Bolivar 542 Huánuco, Perú. Teléfono: (+562) 950871210

Correo electrónico: bernardocristobal@yahoo.com 